\title{
An identification-for-control procedure with robust performance
}

\author{
U. Holmberg*, S. Valentinotti, D. Bonvin \\ Institut d'Automatique, Ecole Polytechnique Fédérale de Lausanne, CH-1015 Lausanne, Switzerland
}

Received 21 July 1998; accepted 27 January 2000

\begin{abstract}
A data-driven controller design procedure is proposed in this paper. The controller is based on both an estimated plant model and its estimated uncertainty described by an ellipsoid in parameter space. Desired performance is specified by the speed and the damping of the modeled response. The unmodeled response is rejected by requiring robust performance with respect to a generalized stability region. Moreover, estimation of a disturbance model enables further rejection of the unmodeled response. The methodology is applied to a nonlinear and unstable magnetic suspension system. High performance is achieved for various specifications over a large operational range. (C) 2000 Elsevier Science Ltd. All rights reserved.
\end{abstract}

Keywords: Robust performance; Least-squares estimation; Control-oriented models; Uncertainty; Magnetic suspension

\section{Introduction}

The concept of identification for control has recently been introduced to emphasize the intended use of the identified model. There have been several attempts to link the identification criterion to the control objective (Bitmead, 1993; Van den Hof \& Schrama, 1995). The identification is typically carried out in a closed loop, since this is the situation the model should be able to describe. For example, if an oscillatory nonlinear system is adequately controlled around a setpoint, a linearized model may suffice to describe the closed-loop behavior. Conversely, the uncontrolled open-loop system may exhibit highly nonlinear responses that cannot adequately be modeled by a linear system. Therefore, the identification of highly oscillatory, unstable and/or nonlinear systems can be expected to benefit greatly from closed-loop identification techniques. Landau and Karimi (1997) illustrated the benefits of using closed-loop identification for a flexible transmission system. Similar benefits were also shown for a highly flexible arm by Langer and Landau (1996). In the latter work, the fact that the controlled plant could handle input signals of larger amplitude was useful to avoid the effects of friction in the

\footnotetext{
* Correspondence address: School of information science, computer and electrical engineering, Halmstad University, Box 823, S-301 18 Halmstad, Sweden. Tel.: + 46-35-167-142; fax: + 46-35-120-348.

E-mail address: ulf.holmberg@ide.hh.se (U. Holmberg).
}

identification procedure. Gevers and Ljung (1986) proved that, for minimum-variance control, closed-loop operation represents an ideal experimental configuration provided that the perfect controller is used (Hjalmarsson, Gevers, De Bruyne \& Leblond, 1996). However it may be argued that, if the perfect controller is already known, there is no incentive for identifying a suitable model for control design. Although the ideal experimental configuration is never achieved in practice, the closed-loop results have been interpreted as one motivation for iterative approaches based on repeated identification and control design (Van den Hof \& Schrama, 1995).

In practice, it is only necessary to re-tune a controller that is not working satisfactorily. The usefulness of iterative schemes can therefore be questioned when convergence is not guaranteed. In order to guarantee improvements from one iteration to the next, De Callafon and Van Den Hof (1997) introduced an iterative scheme based on robust performance. A robust control design based on estimated model uncertainty enables monitoring the control performance before the next iteration is started. However, the procedure, which is formulated in the $H_{\infty}$ framework with identification of the dual Youla parameters, tends to be rather involved and is difficult to implement.

Herein, a different procedure based on robust performance is proposed. Robust performance is specified through the desired speed and damping for an identified uncertainty model set. The model is identified using the 
filtered least-squares method which, in addition to a nominal model, also provides an ellipsoidal parametric uncertainty domain. The model estimation setup is similar to that proposed by Aström (1993) but with the important difference that the data filter and the model uncertainty are estimated. Moreover, designing the controller for robust performance results in substantial performance improvements, thereby avoiding the need for iterations. The procedure proposed is therefore presented as a non-iterative scheme. This paper focuses on the methodology. Thus, the choice of the algorithm used for the robust design problem is considered less important and is not described here.

An optional extension is also proposed for minimizing the unmodeled response when a given reference signal is considered. It is inspired from Tsypkin (1991) (see also the iterative approach by Holmberg, Myszkorowski and Bonvin (1997)) and is based on the internal model principle with the unmodeled response treated as a disturbance. This appears to be more beneficial towards the rejection of the unmodeled response than iterating between model identification and control design.

A magnetic suspension system is chosen to illustrate the methodology. The process represents an excellent benchmark example for identification-for-control schemes since it is both unstable and nonlinear. The scenario considers that a stabilizing but poorly tuned (to motivate re-tuning) controller is available. The objective is to constructively use the measured data for improving the performance, rather than repeating experiments with iterations between identification and controller design. It will also be shown that a tailor-made model that matches the known structures of the plant is quite advantageous for the identification step.

The paper is organized as follows. In Section 2, the robust performance design is presented, consisting of different steps from identification of plant model and uncertainties to robust controller design. The use of the internal model principle for rejecting the unmodeled response is described in Section 3. Section 4 summarizes the methodology in a step-by-step procedure. The magnetic suspension system is described in Section 5, and a tailor-made parameterization is proposed for model estimation. Experimental results are discussed in Section 6 using the proposed procedure with different design specifications. Finally, conclusions are presented in Section 7.

\section{Design for robust performance}

A robust controller is to be designed based on experimental data from a single closed-loop experiment with a stabilizing controller. The procedure involves: (i) identification of a data filter, a model and a model uncertainty set, and (ii) the design of a robust controller. The different steps are described below.

\subsection{Notation}

The plant input, $u(t)$, plant output, $y(t)$, and external reference, $r(t)$, are related via the linear control law

$R(\eta) u(t)=-S(\eta) y(t)+T(\eta) r(t)$.

The plant is, thus, considered to be in closed loop and is modeled as

$A(\theta) y(t)=B_{d}(\theta) u(t)+\varepsilon_{\theta, \eta}(t)$

where $A$ and $B_{d}$ define a linear model. Notice that the equation error, $\varepsilon_{\theta, \eta}(t)$, defined in (2), can be large if the plant is nonlinear or has higher-order dynamics than the linear model. The notation $\varepsilon_{\theta, \eta}(t)$ is used to reinforce the dependence on the plant model parameter vector $\theta$ and the controller parameter vector $\eta$, which are defined as

$\theta=\left(a_{1} \ldots a_{\operatorname{deg} A} b_{d} \ldots b_{\operatorname{deg} B_{d}}\right)^{\mathrm{T}}$,

$\eta=\left(r_{1} \ldots r_{d e g R} s_{0} \ldots s_{d e g s} t_{0}\right)^{\mathrm{T}}$,

where

$$
\begin{aligned}
& A(\theta)=1+a_{1} q^{-1}+\cdots+a_{\operatorname{deg} A} q^{-\operatorname{deg} A}, \\
& B_{d}(\theta)=b_{d} q^{-d}+\cdots+b_{\operatorname{deg} B_{d}} q^{-\operatorname{deg} B_{d}}, \\
& R(\eta)=1+r_{1} q^{-1}+\cdots+r_{\operatorname{deg} R} q^{-\operatorname{deg} R}, \\
& S(\eta)=s_{0}+s_{1} q^{-1}+\cdots+s_{\operatorname{deg} S} q^{-\operatorname{deg} S}, \\
& T(\eta)=t_{0} .
\end{aligned}
$$

All operators above are polynomials in the backwardshift operator $q^{-1}$. The dependencies of the operators and the signals on $q^{-1}$ and $t$, respectively, will be omitted wherever possible for brevity. Combining (1) and (2) and defining

$A_{c}(\theta, \eta)=A(\theta) R(\eta)+B_{d}(\theta) S(\eta)$,

the closed-loop system can be written as

$y=\underbrace{\frac{B_{d}(\theta) T(\eta)}{A_{c}(\theta, \eta)}}_{y_{m}} r+\frac{R(\eta)}{A_{c}(\theta, \eta)} \varepsilon_{\theta, \eta}$.

The term $y_{m}$ is referred to as the modeled closed-loop response, and

$e_{u}=y-y_{m}=\frac{R(\eta)}{A_{c}(\theta, \eta)} \varepsilon_{\theta, \eta}$

is the unmodeled response that is driven by unmodeled dynamics and external disturbances via $\varepsilon_{\theta, \eta}$.

\subsection{Identification of a data filter and a plant model}

Suppose that $N$ data points have been collected using a stabilizing controller with the parameters $\eta_{i}$. From (2) and (5), and using a stable filter $W$ chosen such that $W R\left(\eta_{i}\right)=\left(1-q^{-1}\right)$ to eliminate nonzero-mean data 
(e.g. if the controller lacks integral action), the filtered unmodeled response can be expressed as

$W e_{u}(t, \theta)=A(\theta) y_{F}(t)-B_{d}(\theta) u_{F}(t)=y_{F}(t)-\varphi(t)^{\mathrm{T}} \theta$,

where

$$
\begin{aligned}
\varphi(t)= & \left.\mid \begin{array}{c}
-y_{F}(t-1) \\
\cdots \\
-y_{F}(t-\operatorname{deg} A) \\
u_{F}(t-d) \\
\cdots \\
u_{F}\left(t-\operatorname{deg} B_{d}\right)
\end{array}\right), \\
& \left\{\begin{array}{l}
y_{F}(t)=F y(t), \\
u_{F}(t)=F u(t),
\end{array} \quad F\left(\theta, \eta_{i}\right)=\frac{1-q^{-1}}{A_{c}\left(\theta, \eta_{i}\right)} .\right.
\end{aligned}
$$

At first glance, it appears that the model estimation can be achieved using the linear least-squares method, i.e., the criterion

$$
V\left(\theta, \eta_{i}\right)=\frac{1}{N} \sum_{t=1}^{N}\left[W e_{u}(t, \theta)\right]^{2}=\frac{1}{N} \sum_{t=1}^{N}\left[y_{F}(t)-\varphi(t)^{\mathrm{T}} \theta\right]^{2} .
$$

However, the linear dependence on $\theta$ in (6) is deceptive since the data filter $F$ also depends on $\theta$. (Åström, 1993) ignored the nonlinear dependence by replacing $A_{c}\left(\theta, \eta_{i}\right)$ in $F$ by $A_{c}^{D}$ chosen as a design variable in a poleplacement approach. This is not necessarily adequate. To clarify this point, the notation $e_{u, N L}\left(\theta, \eta_{i}\right)$ is introduced to emphasize the nonlinear dependence on $\theta$ via $F\left(\theta, \eta_{i}\right)$. Furthermore, $e_{u, L}\left(\theta, \eta_{i}\right)$ denotes the corresponding linear expression where the data filter is fixed, $F^{D}=$ $\left(1-q^{-1}\right) / \boldsymbol{A}_{c}^{D}$. The nonlinear criterion (7) is $V_{N L}\left(\theta, \eta_{i}\right)=$ $\left\|W e_{u, N L}\right\|$. The modified linear criterion is related to $W e_{u, N L}$ as

$V_{L}\left(\theta, \eta_{i}\right)=\left\|W e_{u, L}\right\|=\left\|\frac{A_{c}\left(\theta, \eta_{i}\right)}{A_{c}^{D}} W e_{u, N L}\right\|$.

This suggests that, when the linear criterion is used to find an estimate $\hat{\theta}$, closeness of $A_{c}\left(\hat{\theta}, \eta_{i}\right)$ and $A_{c}^{D}$ should be checked to justify the linear problem formulation. Continuing this argument, just as $A_{c}\left(\hat{\theta}, \eta_{i}\right)$ represents an estimate of the closed-loop characteristic polynomial, it would be judicious to select $A_{c}^{D}$ as an educated guess of that polynomial as well. This can be done by choosing it from an estimate of the closed-loop system from $r$ to $y$. Introduce

$A_{c}^{*}\left(\theta_{c}, \eta_{i}\right)=1+a_{c 1} q^{-1}+\cdots+a_{c \operatorname{deg} A_{c}^{*}} q^{-\operatorname{deg} A_{c}^{*}}$,

where $\theta_{c}=\left(a_{c 1} \cdots a_{c \operatorname{deg} A_{c}^{*}}\right)^{\mathrm{T}}$. The choice of $A_{c}^{D}$ is replaced by an estimation problem for $\theta_{c}$. Standard prediction error methods can be used. From (4), an ARMAX structure seems appropriate.
Thus, in order to make $V_{L}\left(\theta, \eta_{i}\right)$ close to $V_{N L}\left(\theta, \eta_{i}\right)$, the data filter is chosen as

$F^{*}=\frac{1-q^{-1}}{A_{c}^{*}\left(\hat{\theta}_{c}, \eta_{i}\right)}$.

Then, with the criterion $V_{L}\left(\theta, \eta_{i}\right)$, the parameter estimation becomes a linear least-squares problem with the analytical solution

$\widehat{\theta}=\left[\sum_{t=1}^{N} \varphi(t) \varphi(t)^{\mathrm{T}}\right]^{-1} \sum_{t=1}^{N} \varphi(t) y_{F^{*}}(t)$.

Clearly, the criterion $V_{L}\left(\theta, \eta_{i}\right)$ and the estimates depend on the initial controller $\eta_{i}$ via $u$ and $y$. The choice of a data filter also suggests validating the model prior to controller implementation. The way that this can be performed is described below.

\subsection{Validation of the data filter and the plant model}

The linear least-squares problem, resulting from keeping the data filter $F^{*}$ fixed, is a valid approximation to the original nonlinear problem provided that the polynomials $A_{c}\left(\hat{\theta}, \eta_{i}\right)$ and $A_{c}^{*}\left(\hat{\theta}_{c}, \eta_{i}\right)$ are 'close' in some sense. In order to introduce a measure of 'closeness' between (characteristic) polynomials, define $\lambda\left(A_{c}\right)=$ $\left\{z: A_{c}\left(z^{-1}\right)=0\right\}$ (which are the reciprocal poles of the system, or equivalently, the poles of the system when using the forward-shift operator). Those $z \in \lambda\left(A_{c}\right)$ closest to the unit circle have the most dominant influence on the response. Hence, an a priori (prior to implementation) validation test is to compare $\lambda\left(A_{c}\left(\hat{\theta}, \eta_{i}\right)\right)$ and $\lambda\left(A_{c}^{*}\left(\hat{\theta}_{c}, \eta_{i}\right)\right)$ for $|z|$ closest to one.

Note that since both $B_{d}\left(\theta_{c}\right)$ and $A_{c}^{*}\left(\theta_{c}, \eta_{i}\right)$ can be estimated as the closed-loop dynamics from $r$ to $y$, an estimate of $A(\theta)$ could be calculated from relation (3). But this is inconvenient since it requires a polynomial division, which, furthermore, makes it difficult to obtain uncertainty bounds.

\subsection{Estimation of uncertainty bounds}

Since the least-squares method is used in the model identification, it is natural to take advantage of the ellipsoidal parameter error bound associated with it. A bound on the estimation error, $\Delta \theta=\hat{\theta}-\theta_{\boldsymbol{P}}$, where $\theta_{\boldsymbol{P}}$ is an unknown 'true' parameter value, can be expressed by an ellipsoid (Ljung, 1987)

$\Delta \theta^{\mathrm{T}} \mathbf{P}^{-1} \Delta \theta \leq 1$.

For example, a $100 \alpha \%$ confidence ellipsoid is obtained with

$\mathbf{P}=\chi_{n, \alpha}^{2} \frac{N}{N-n} V\left(\hat{\theta}, \eta_{i}\right)\left[\sum_{t=1}^{N} \varphi(t) \varphi(t)^{\mathrm{T}}\right]^{-1}$, 
where $n$ is the dimension of $\theta$, and $\chi_{n, \alpha}^{2}$ the $\alpha$-level of the $\chi^{2}$ distribution with $n$ degrees of freedom. The derivation of this bound is based on the assumption that $W e_{u}$ is white noise, which will never be achieved exactly. Nonetheless, it still makes sense to use this uncertainty description to improve the robustness of the controller.

\subsection{Robust controller design}

The control objective is to make the dynamics from $r$ to $y$ well behaved in a certain sense, e.g. having a specified speed and damping. For the continuous-time system $G(s)=\omega_{0}^{2} /\left(s^{2}+2 \zeta \omega_{0} s+\omega_{0}^{2}\right)$, with poles $p_{1,2}=$ $-\zeta \omega_{0} \pm \mathrm{i} \omega_{0} \sqrt{1-\zeta^{2}}$, the step response speed and overshoot are solely determined by $\omega_{0}$ and $\zeta$, respectively. The larger $\omega_{0}$ the faster the response, and the smaller $\zeta(0<\zeta<1)$ the larger the overshoot. Therefore, standard pole placement design could be used for assessing the speed and damping of the dynamics from $r$ to $y_{m}$. However, this gives no guarantee that the contribution of the unmodeled response $e_{u}=y-y_{m}$ is small and, consequently, the response $y$ may deteriorate due to disturbances. To circumvent this problem, a robust approach is taken. It can be formulated as follows:

Find a controller $\eta$ such that $\lambda\left(A_{c}(\theta, \eta)\right) \in D$ for all $\theta$ in the ellipsoid $(\hat{\theta}-\theta)^{\mathrm{T}} \mathbf{P}^{-1}(\hat{\theta}-\theta) \leq 1$, where $D$ represents the desired stability region.

\subsubsection{Parameterization of the desired stability region}

The generalized stability region $D$ is chosen to be a subset of the unit disc. For simplicity, the boundary of the $D$ region, symmetrical around the real axis, can be parameterized as $z=\mathrm{e}^{\mathrm{i} \omega} \kappa(\omega), \omega \in[0, \pi]$ where

$\kappa(\omega)= \begin{cases}\mathrm{e}^{-\sqrt{\omega_{\text {min }}^{2}-\omega^{2}}}, & 0 \leq \omega \leq \omega_{\min } \sqrt{1-\zeta_{\text {min }}^{2}}, \\ \mathrm{e}^{-\frac{\zeta_{\min }}{{\sqrt{1-\zeta^{2} \min }}^{2}},}, & \omega_{\min } \sqrt{1-\zeta_{\min }^{2}}<\omega \leq \pi .\end{cases}$

This means that, for any $z \in D$, with corresponding natural frequency $\omega_{0}$ (in radians per number of sampling periods) and relative damping $\zeta$, defined as $z=\mathrm{e}^{s}$ with $s=-\zeta \omega_{0}+\mathrm{i} \omega_{0} \sqrt{1-\zeta^{2}}$, it follows that $\omega_{0}>\omega_{\min }$ and $\zeta>\zeta_{\min }$. Thus, $\omega_{\min }$ and $\zeta_{\min }$ specify the position of the dominant (forward-shift) poles (those $|z|$ closest to one). The design parameters for the $D$ region, $\omega_{\min }$ and $\zeta_{\min }$, do not correspond exactly to the speed and overshoot, since the influence of both the nondominant poles and the zeros have been neglected. The shape of $D$ is therefore not 'holy' and the suggested parameterization is used only for simplicity. Examples of $D$ regions are given in Figs. $3 \mathrm{~b}$ and $5 b$.

\subsubsection{Fixed factors in $R$ and $S$}

It is usually of interest to specify the fixed factors $R_{f i x}$ and $S_{f i x}$ of the polynomials $R$ and $S$, respectively, as constraints in the robust design problem.
For example, the choice $R_{f i x}=1-q^{-1}$ gives integral action to the controller and the choice $S_{f i x}=1+q^{-1}$ reduces high-frequency gain and, therefore, the sensitivity to measurement noise.

\subsubsection{Algorithms for the robust controller synthesis}

Various algorithms can be used to solve the robust control problem formulated above. It is not the purpose of this paper to describe them in detail. Instead the focus is on the methodology for using robust control techniques in an identification-for-control procedure. The particular algorithm for robust control design used here was the pole-projection approach proposed by Holmberg and Valentinotti (1997). This was chosen because of its algorithmic simplicity and its flexibility regarding the choice of the $D$ region. Furthermore, the pole-projection approach does not approximate the problem and may therefore be less conservative than other approaches such as those proposed by Rantzer and Megretski (1994) and Raynaud (1991). The main drawback with the approach by Rantzer and Megretski (1994) is that the controller is found numerically by searching in an infinite-dimensional space, and any reasonable truncation gives impractically high-order controllers. The $H_{\infty}$ approach by Raynaud (1991) gives a finite-order controller of reasonable order, but is restricted to $D$ regions of simple shapes such as circles.

\section{Minimizing the unmodeled response}

The unmodeled response is made small by the specification of robust performance. For further reduction of the unmodeled response, the difference in closed-loop response between the real plant and the nominal model can be used. The benefit with this approach is that the unmodeled response can be rejected considerably, in particular when only one specific reference signal is used, making the model mismatch dependent on that reference. The drawbacks are that a new experiment should be made with a robust controller and the complexity of the controller increases. The key idea is to include a disturbance model in the controller according to the classical concept of internal model principle. Since the disturbance model serves to describe the unmodeled response, it is unknown a priori. However, using an appropriate parameterization of the controller, the disturbance model can be estimated using linear least-squares estimation.

\subsection{Internal model principle}

Suppose the disturbance $\varepsilon_{\theta, \eta}$ can be modeled as $\Phi \varepsilon_{\theta, \eta}(t)=0, t \geq \operatorname{deg} \Phi\left(q^{-1}\right)$, where $\Phi$ is a known polynomial. Many disturbances can be modeled in this way. For example, the class of steps or offsets of any size corresponds to $\Phi\left(q^{-1}\right)=1-q^{-1}$; ramps are modeled 
by $\Phi=\left(1-q^{-1}\right)^{2} ; N$-periodic signal are modeled by $\Phi=1-q^{-N}$, etc. The notion of internal model principle is to include this disturbance model in the controller by requiring $\Phi$ to be a factor in $R$. Then, the unmodeled response $e_{u}=\left[R(\eta) / A_{c}(\theta, \eta)\right] \varepsilon_{\theta, \eta} \rightarrow 0$ for $t \rightarrow \infty$.

\subsection{Estimation of the internal model}

The concept can be generalized to be used even when the polynomial $\Phi$ is partially or completely unknown, which is the situation considered here. The equation error $\varepsilon_{\theta, \eta}$ will, due to imperfect modeling, include unknown parts from the model mismatch. Rather than trying to estimate directly $\Phi$, an indirect parameterization is used that enables, as before, the estimation to be posed as a linear least-squares problem.

Parameterization of all solutions $\{R, S\}$, including the pre-chosen factors $R_{f i x}, S_{f i x}$, to the polynomial equation (3) gives

$$
\begin{aligned}
& R\left(\eta\left(\theta_{Q}, \theta\right)\right)=\left(R_{1}-Q\left(\theta_{Q}\right) B_{\mathrm{d}}(\theta) S_{f i x}\right) R_{f i x}, \\
& S\left(\eta\left(\theta_{Q}, \theta\right)\right)=\left(S_{1}+Q\left(\theta_{Q}\right) A(\theta) R_{f i x}\right) S_{f i x},
\end{aligned}
$$

where $\left\{R_{1} R_{f i x}, S_{1} S_{f i x}\right\}$ is one particular solution and $Q$ is an arbitrary polynomial. The unmodeled response $e_{u}$ defined in (5) can be parameterized using the polynomial $Q\left(q^{-1}\right)=q_{0}+q_{1} q^{-1}+\cdots+q_{\text {deg } Q} q^{-\operatorname{deg} Q}$ as

$e_{u}\left(t, \theta_{Q}\right)=\varepsilon_{1}(t)-Q \varepsilon_{2}(t)=\varepsilon_{1}(t)-\varphi_{\varepsilon}(t)^{\mathrm{T}} \theta_{Q}$,

where $\theta_{Q}=\left(q_{0} \ldots q_{\operatorname{deg} Q}\right), \varphi_{\varepsilon}(t)=\left(\varepsilon_{2}(t) \ldots \varepsilon_{2}(t-\operatorname{deg} Q)\right)$ and

$\varepsilon_{1}=\frac{R_{1} R_{f i x}}{A_{c}(\theta, \eta)} \varepsilon_{\theta, \eta}$,

$\varepsilon_{2}=\frac{B_{\mathrm{d}} S_{f i x} R_{f i x}}{A_{c}(\theta, \eta)} \varepsilon_{\theta, \eta}$.

Thus, for an estimated plant model and a corresponding $A_{c}$, the unmodeled response $e_{u}\left(t, \theta_{Q}\right)$ becomes linear in $\theta_{Q}$. Identification of $\theta_{Q}$ can therefore be posed as a linear least-squares problem with the criterion

$V_{\theta}\left(\theta_{Q}\right)=\sum_{t=1}^{N} e_{u}\left(t, \theta_{Q}\right)^{2}=\sum_{t=1}^{N}\left[\varepsilon_{1}(t)-\varphi_{\varepsilon}(t)^{\mathrm{T}} \theta_{Q}\right]^{2}$,

having the minimizing solution $\hat{\theta}_{Q}=$ $\left[\sum_{t=1}^{N} \varphi_{\varepsilon}(t) \varphi_{\varepsilon}(t)^{\mathrm{T}}\right]^{-1} \sum_{t=1}^{N} \varphi_{\varepsilon}(t) \varepsilon_{1}(t)$. According to (12), the controller parameters $\eta$ are linear functions of the polynomial $Q$, which is used to indirectly model the disturbance, i.e., to obtain the appropriate factor $\Phi$ in $R$ according to the internal model principle. If no a priori assumption is made about $\Phi$, the choice $R_{f i x}=1$ is made in (12).

Notice that, since unmodeled dynamics can give a destabilizing controller, this approach should only be applied after a robust pole placement has been found. Thus, a new experiment using the robust controller should be made for the estimation of $Q$.

A particular feature with this approach is that the number of parameters to be estimated $(\operatorname{deg} Q)$ can be chosen freely and, therefore, can be smaller than is needed for estimating the plant model. This is of particular importance in cases of small signal-to-noise ratio when reliable estimation of many parameters is not possible. In other words, the fact that fewer parameters are estimated enables the use, in the identification experiment, of excitation signals that are used in normal operation. This is beneficial for optimal disturbance rejection for normal operation.

\section{Identification-for-control procedure}

The proposed identification-for-control procedure that uses a single data set is summarized below in Steps 1-7. Optionally, Steps 8-9 allow further minimization of the unmodeled response for a particular reference signal, but at the price of a repeated experiment and a more complex controller.

1. Collect $N$ data points of $(r, y, u)$ in closed-loop operation, corresponding to deviations from the setpoint $\left(r_{0}, y_{0}, u_{0}\right)$ using the (stabilizing) controller $\eta_{i}$.

2. Estimate $\hat{\theta}_{c}$ using a simple ARX or ARMAX structure and construct the data filter $F^{*}=\left(1-q^{-1}\right) / A_{c}^{*}\left(\hat{\theta}_{c}, \eta_{i}\right)$.

3. Compute the filtered least-squares estimate $\hat{\theta}$ as in (9).

4. Compare the dominant (close to unit circle) poles of $\lambda\left(A_{c}\left(\hat{\theta}, \eta_{i}\right)\right)$ with those of $\lambda\left(A_{c}^{*}\left(\hat{\theta}_{c}, \eta_{i}\right)\right)$. Continue to next step if a close fit is found, otherwise repeat Step 2 with a different model structure.

5. Choose a level of confidence and calculate the ellipsoidal uncertainty set (10).

6. Choose the desired performance by specifying $\omega_{\min }$ and $\zeta_{\text {min }}$.

7. Specify the fixed factors $R_{f i x}$ and $S_{f i x}$ and solve the robust control design problem by any suitable method (see Section 2.5) to obtain $\eta(\widehat{\theta}, \mathbf{P})$. If no solution is found, either decrease the level of confidence (i.e. the size of the ellipsoidal uncertainty set) in Step 5 or relax the performance specifications in Step 6.

8. Repeat Steps $1-4$ using the robust controller $\eta(\hat{\theta}, \mathbf{P})$ from Step 7

9. Choose $R_{f i x}, S_{f i x}, \operatorname{deg} Q$ and calculate the leastsquares estimate $\hat{\theta}_{Q}$ using the model (13), whereafter the corresponding controller follows from (12).

\section{Magnetic suspension system}

The procedure above will now be illustrated for a laboratory system. First, the nonlinear and unstable magnetic suspension system is described. It is then shown 
a)

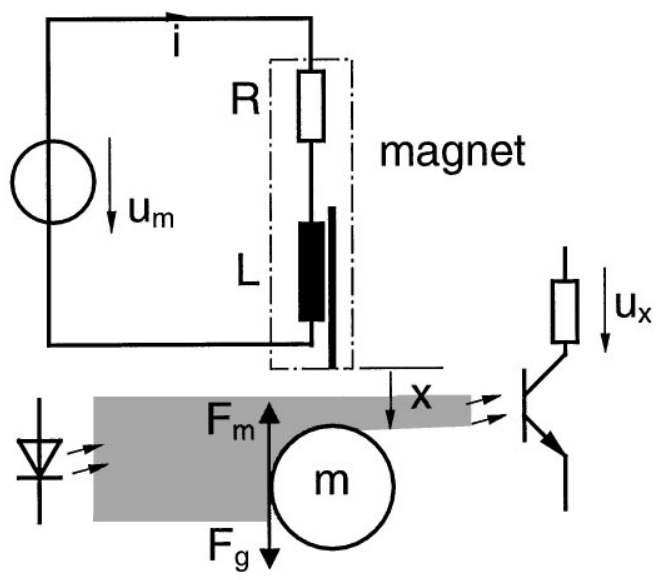

b)

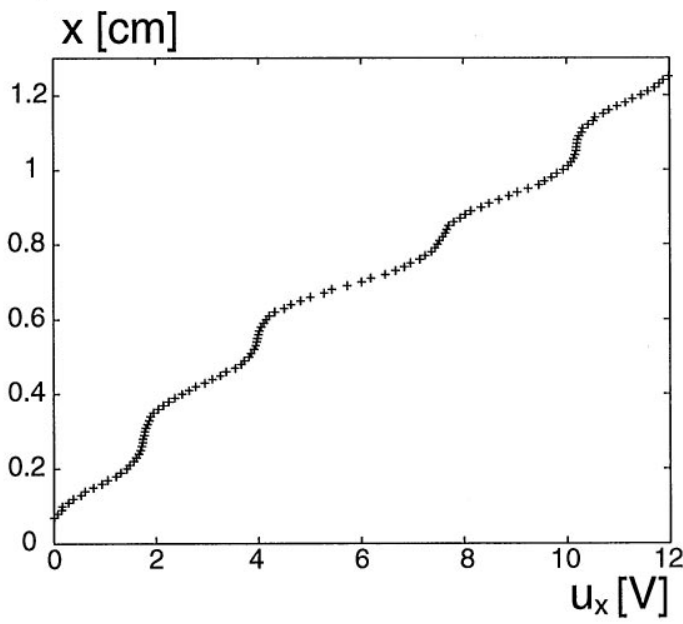

Fig. 1. (a) Magnetic suspension system. (b) Nonlinear sensor characteristics.

how structural knowledge can be taken into account to reduce the number of parameters to be estimated. The experimental results are given in Section 6.

\subsection{Experimental setup}

The magnetic suspension system consists of keeping an iron ball suspended in the air by using a magnetic force to compensate for the gravitational force. A schematic description of the process is given in Fig. 1a. A force balance gives the equation

$m \ddot{x}=F_{g}-F_{m}$,

where $m$ is the mass of the ball, $x$ its position, $F_{g}=m g$ the gravitational force and $F_{m}$ is the magnetic force. The latter can be expressed as $F_{m}=-\frac{1}{2}(\mathrm{~d} L / \mathrm{d} x) i^{2}$, where $L$ is the inductance that decreases with $x$. The dynamics of the current $i$ can be approximated well by a first-order system. The current loop is controlled by an analog high-gain controller and, therefore, its dynamics can be neglected. Since $\mathrm{d} L / \mathrm{d} x$ depends on $x$, linearization of the ball dynamics around a stationary point gives the following transfer function between the magnet voltage $u_{m}$ and the sensor voltage $u_{x}$ :

$G(s)=\frac{b}{s^{2}-a}$.

The parameters $a$ and $b$ vary with the linearization point, especially $b$ due to the particular nonlinear relationship between the measured voltage $u_{x}$ and the ball position $x$ (Fig. 1b). Sampling using zero-order hold gives the discrete-time system

$H\left(q^{-1}\right)=\gamma \frac{q^{-1}+q^{-2}}{1-\beta q^{-1}+q^{-2}}, \quad \beta>0$.
Thus, there are only two unknown parameters to be estimated rather than the four of a general second-order system.

\subsection{Scales and units}

Engineers like to display input and output signals as physically meaningful-quantities. This makes sense. However, a common mistake is to use these signals without proper scaling directly in the identification algorithm. The problem comes from the fact that unscaled signals might be of different orders of magnitude, leading to parameters of different orders of magnitude as well. Thus, the variance of the larger parameters will totally destroy the accuracy of the smaller ones. The problem is usually avoided if the signals are scaled appropriately, i.e., each scaled signal covers the same domain (e.g. $-1-1$, or $0-1)$. Alternatively, the signals can be taken directly, without scaling, from the $\mathrm{A} / \mathrm{D}$ and $\mathrm{D} / \mathrm{A}$ converters. For the magnetic suspension system, the input $u$ corresponds to the $\mathrm{D} / \mathrm{A}$ converter voltage deviation from the linearization point $u_{0}$. The output $y$ is the measured $\mathrm{A} / \mathrm{D}$ converted voltage $u_{x}$ (Fig. 1a) calibrated through the static sensor nonlinearity to correspond to the ball distance deviation. The calibration serves to compensate for the worm-like shape of the sensor characteristics and not to introduce a scale that changes the magnitude. The resulting unit for $y$ is $10^{-4} \mathrm{~m}$.

\subsection{Tailor-made plant model estimation}

From (6) and (14), the unmodeled response can be parameterized as follows:

$W e_{u}(t, \theta)=z(t)-\varphi(t)^{\mathrm{T}} \theta$, 
where

$\theta=\left(\begin{array}{ll}\beta & \gamma\end{array}\right)^{\mathrm{T}}$,

$z(t)=\left(1+q^{-2}\right) y_{F^{*}}(t)$,

$\varphi(t)=\left[q^{-1} y_{F^{*}}(t)\left(q^{-1}+q^{-2}\right) u_{F^{*}}(t)\right]^{\mathrm{T}}$,

and $y_{F^{*}}=F^{*} y \quad$ and $\quad u_{F^{*}}=F^{*} u$ with $F^{*}=$ $\left(1-q^{-1}\right) / A_{c}^{*}\left(\hat{\theta}_{c}, \eta_{i}\right)$. Least-squares estimation gives $\hat{\theta}=$ $\left[\sum_{t=1}^{N} \varphi(t) \varphi(t)^{\mathrm{T}}\right]^{-1} \sum_{t=1}^{N} \varphi(t) z(t)$, and the uncertainty domain (10) is an ellipse.

\section{Experimental results}

First, following Steps 1-4 of the procedure given in Section 4, the closed-loop and open-loop models will be estimated and validated. Then, continuing with Steps $5-7$, various design choices will be discussed. These include the size of the uncertainty ellipse, the desired response speed, and trading off the size of the unmodeled response to reduce control effort. Finally, after a second experiment using the robust controller from Step 7, Steps 8 and 9 illustrate the improvement obtained by using the internal model principle to reject the unmodeled response.

\subsection{Plant model estimation}

Step 1: Collecting the initial closed-loop response

The initial parameter vector $\eta_{i}=(-0.110 .1344$ - 0.0910 .0499$)(\operatorname{deg} R=1, \operatorname{deg} S=1)$ gives a PD-like controller. The stationary linearization point $u_{0}$ is manually adjusted to make $y_{0}$ approach $r_{0}$ without static offset. A square wave excitation of the reference deviation $r$ is then chosen with an amplitude of $1.5 \times 10^{-4} \mathrm{~m}$. Larger amplitudes are not possible due to saturation of the control input $u$. The total data length used for estimation is $5 \mathrm{~s}$. Three seconds of the initial closed-loop response are shown in Fig. 2.

Steps 2-4: Data filter estimation using an ARX structure

There is no need to choose the degrees of $\left.A_{c}^{*}\left(\hat{\theta}_{c}, \eta_{i}\right)\right)$ and $\left.A_{c}\left(\hat{\theta}, \eta_{i}\right)\right)$ the same since the latter includes nondominant poles (close to the origin). By choosing $\operatorname{deg} A_{c}^{*}\left(\hat{\theta}_{c}, \eta_{i}\right)$ smaller to only include the dominant part, the identification is easier with less requirement on the excitation. For simplicity, an ARX structure with $\operatorname{deg} A_{c}^{*}\left(\hat{\theta}_{c}, \eta_{i}\right)=2$ is tried first. The calculated and the directly estimated closed-loop poles are

$$
\begin{aligned}
& \lambda\left(A_{c}\left(\hat{\theta}, \eta_{i}\right)\right)=0.8663 \pm 0.2771 i, 0.2604, \\
& \lambda\left(A_{c}^{*}\left(\hat{\theta}_{c}, \eta_{i}\right)\right)=0.7622 \pm 0.2458 i .
\end{aligned}
$$

The dominant poles are rather close. Nevertheless, it may be of interest to see whether closer fit can be obtained a) $\mathrm{y}, \mathrm{r}\left[10^{-4} \mathrm{~m}\right]$
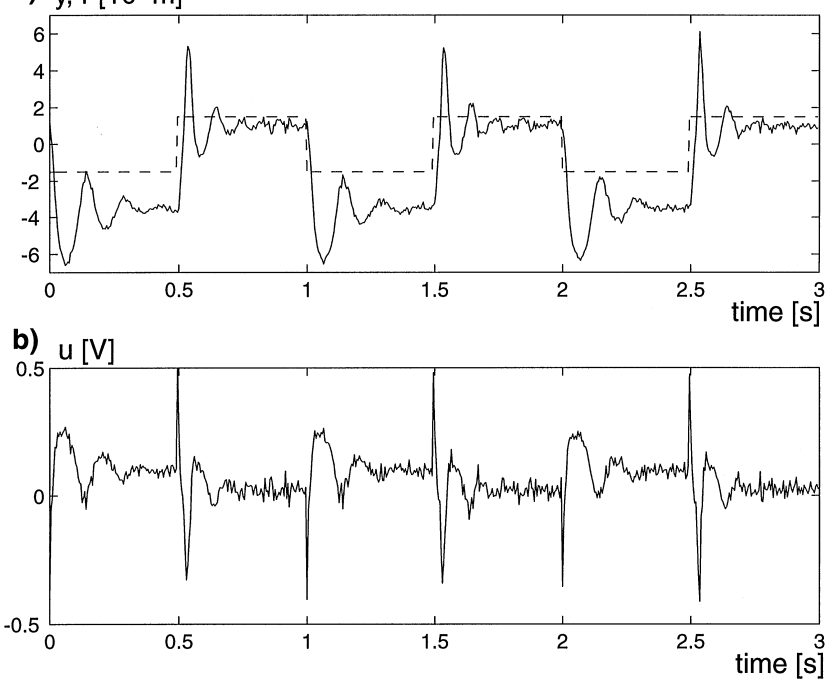

Fig. 2. Initial closed-loop response. (a) $y$ (solid), $r$ (dashed); (b) $u$.

with a data filter estimated using an ARMAX structure which, according to (4), should be more appropriate.

Steps 2-4 repeated: Data filter estimation using an ARMAX structure

With an ARMAX structure,

$$
\begin{aligned}
& \lambda\left(A_{c}\left(\hat{\theta}, \eta_{i}\right)\right)=0.8900 \pm 0.2600 i, 0.2300, \\
& \lambda\left(A_{c}^{*}\left(\hat{\theta}_{c}, \eta_{i}\right)\right)=0.8821 \pm 0.2554 i .
\end{aligned}
$$

The dominant closed-loop poles are closer, which improves the confidence in the estimated model. The parameter vector estimated in Step 3 is

$\hat{\theta}=(\widehat{\beta} \hat{\gamma})^{\mathrm{T}}=\left(\begin{array}{lll}2.0203 & 0.9217\end{array}\right)^{\mathrm{T}}$.

\subsection{Slow desired response with large robustness}

Step 5: Uncertainty ellipse specification

The $\mathbf{P}$ matrix in (11) is scaled to correspond to a $99 \%$ confidence level. The resulting large ellipse will thus account for large uncertainties, see Fig. 3a.

Step 6: Performance specifications

The performance is specified by the choice of the generalized stability region, $D$. It is characterized by $\omega_{\min }=0.05$ (slow response) and $\zeta_{\min }=0.7$ (well-damped response), see Fig. 3b.

Step 7: Robust controller synthesis

Integral action is specified by choosing $R_{f i x}=$ $1-q^{-1}$. The resulting pole placement satisfying robust performance is

$\lambda\left(A_{c}(\hat{\theta}, \eta(\hat{\theta}, \mathbf{P}))\right)=0.9465,0.5884,0.4220,0.1528$.

The corresponding closed-loop response is shown in Fig. 4. Notice the remarkably close fit between the 

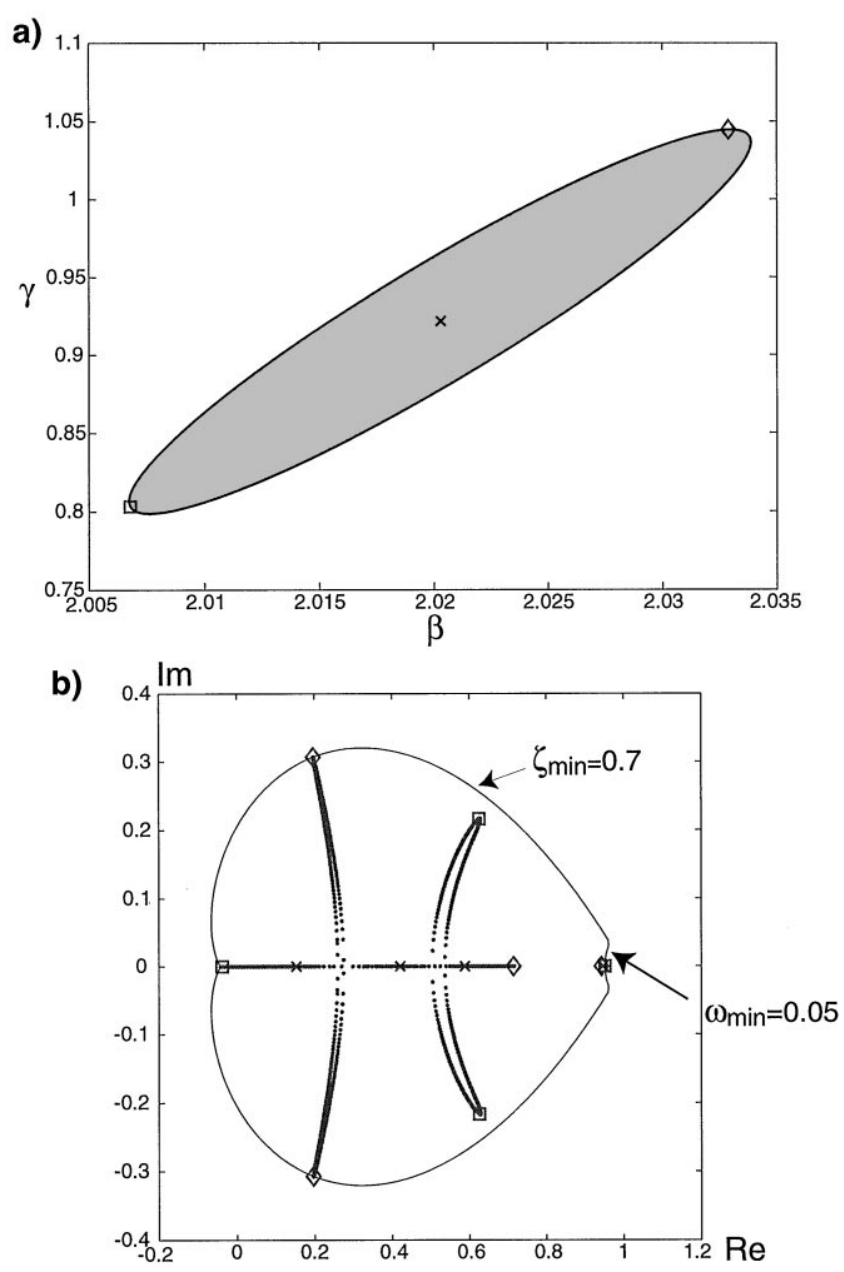

Fig. 3. Robust design for slow response. (a) Nominal model ' $x$ ' and $99 \%$ uncertainty ellipse; (b) generalized stability region $\left(\omega_{\min }=0.05\right.$, $\left.\zeta_{\min }=0.7\right)$. Closed-loop nominal poles ' $x$ ' and poles corresponding to the ellipse boundary (with special examples marked $\square$ and $\diamond$ ).

modeled response $y_{m}$ (dashed) and the actual response $y$ (solid). As a posteriori validation of the scheme, the unmodeled response standard deviation is evaluated: $\operatorname{STD}\left(e_{u}\right)=0.10 \times 10^{-4} \mathrm{~m}$ and the closed-loop poles re-estimated

$\lambda\left(A_{c}^{*}\left(\widehat{\theta}_{c}, \eta(\hat{\theta}, \mathbf{P})\right)\right)=0.9512,0.5313$.

The modeled response $y_{m}$ is mostly dependent on the dominant slow pole near 0.95 . However, if $A_{c}^{D}=$ $1-0.95 q^{-1}$ would be chosen in a standard pole placement design, the resulting controller would not satisfy robust performance, and in fact, would not stabilize the real system. Conversely, the robust design makes all the plants in the estimated uncertainty set satisfy the performance specification. Moreover, the dominant closedloop poles are almost the same for all the plants in the uncertainty set (Fig. 3b) which is the reason for the invariant, linear-like closed-loop behavior (compare Figs. 2 and 4).
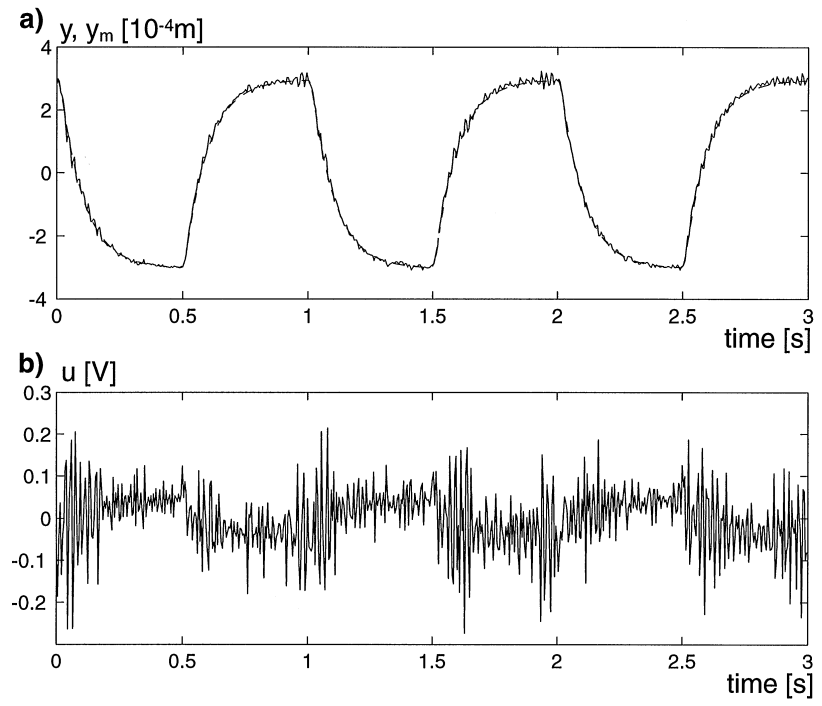

Fig. 4. Robust design for slow response. (a) $y$ (solid), $y_{m}$ (dashed); (b) $u$.

\subsection{Trade-off between unmodeled response and control effort}

The small variance in the unmodeled response error is at the cost of a very noisy control signal. Quantification noise caused by the finite precision in the $\mathrm{A} / \mathrm{D}$ converter may be one significant noise source. Let the noise level in the control signal be measured as $\max |\Delta u|$, with $\Delta u$ being the change of $u$ between two consecutive samples. This noise level can be reduced by choosing $S_{f i x}=1+q^{-1}$, which makes the controller gain drop to zero at the Nyquist frequency. This modification reduces the noise level in the control signal by a factor of nearly 4 at the price of an increase in the unmodeled response standard deviation by a factor of 1.5 (see Table 1).

\subsection{Faster desired response with lesser robustness}

Step 5: Uncertainty ellipse

The uncertainty ellipse is now reduced to correspond to a $90 \%$ confidence level in order to make it possible to obtain robust performance with a smaller $D$ region, see Figs. $5 \mathrm{a}$ and $\mathrm{b}$.

Step 6: Performance specification

A faster response is specified by choosing $\omega_{\min }=0.2$, see Fig. $5 b$.

Step 7: Robust controller synthesis

In order to avoid control signal saturation and noise amplification, $\boldsymbol{S}_{f i x}=1+q^{-1}$ is chosen. The pre-chosen factor in $R$ is specified to be $R_{f i x}=1-0.96 q^{-1}$, rather than, as before, $\boldsymbol{R}_{f i x}=1-q^{-1}$. This modification reduces the effect of high-frequency disturbances on the unmodeled response. The static offset is removed by manual 
Table 1

Noise reduction in the control signal by using $S_{f i x}=1+q^{-1}$

\begin{tabular}{lll}
\hline$S_{f i x}$ & $\operatorname{STD}\left(e_{u}\right)\left(10^{-4} \mathrm{~m}\right)$ & $\operatorname{Max}|\Delta u|(\mathrm{V})$ \\
\hline 1 & 0.10 & 0.45 \\
$1+q^{-1}$ & 0.15 & 0.12
\end{tabular}
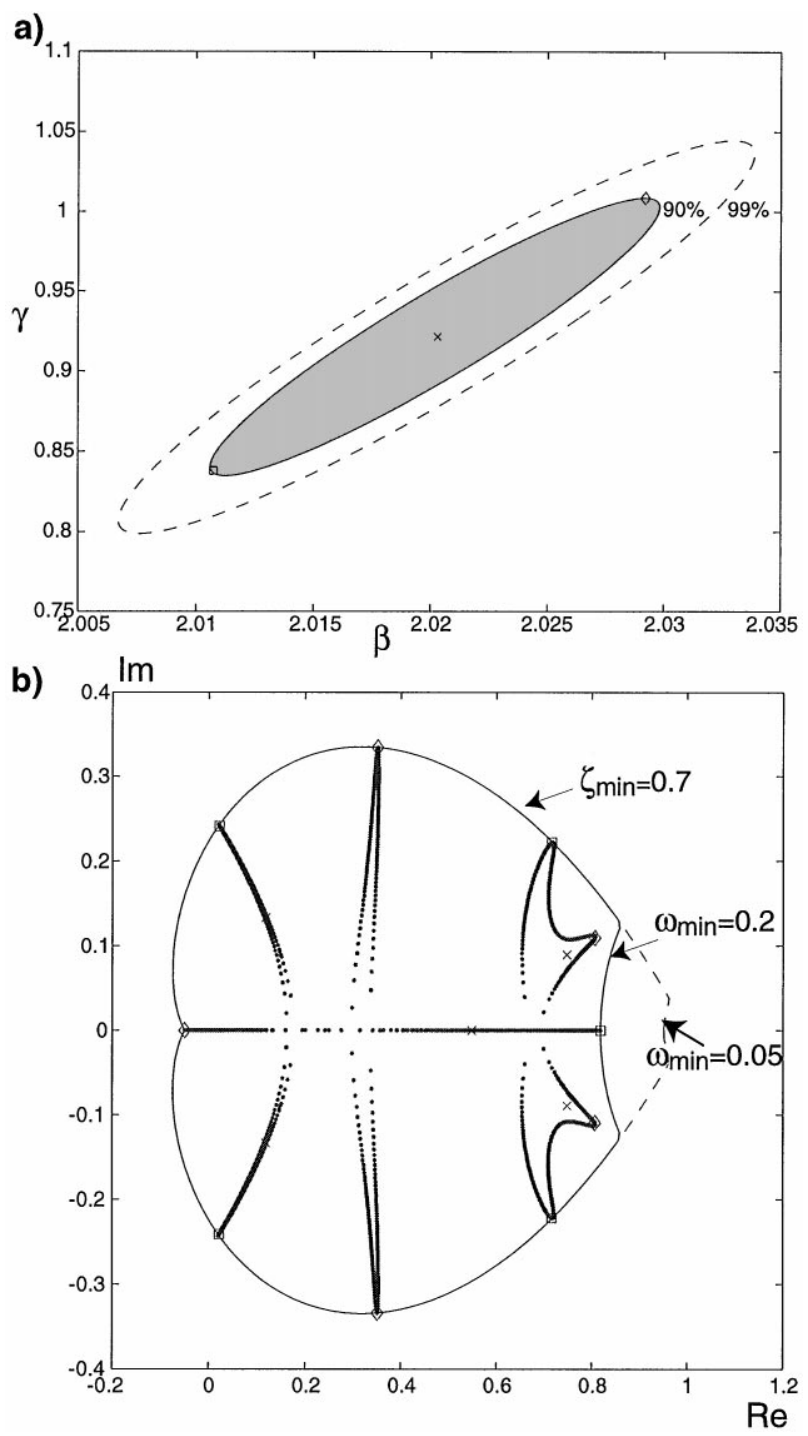

Fig. 5. Robust design for faster response. (a) Nominal model ' $x$ ' and $90 \%$ uncertainty ellipse; (b) generalized stability region $\left(\omega_{\min }=0.2\right.$, $\zeta_{\min }=0.7$ ). Closed-loop nominal poles ' $x$ ' and poles corresponding to the ellipse boundary (with special examples marked $\square$ and $\diamond$ ).

tuning of $u_{0}$. The resulting pole placement satisfying robust performance is

$$
\begin{aligned}
\lambda\left(A_{c}(\hat{\theta}, \eta(\hat{\theta}, \mathbf{P}))\right)= & 0.7603 \pm 0.0954 i, 0.5416,0.1201 \\
& \pm 0.1312 i .
\end{aligned}
$$

From Fig. 5b, it is seen that the dominant closed-loop poles for various plants in the uncertainty ellipse can be
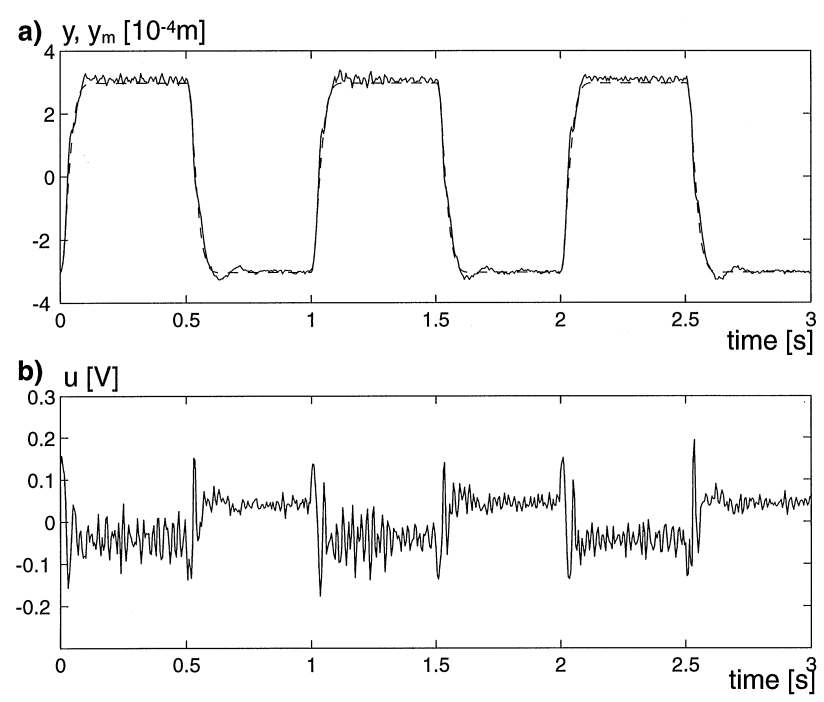

Fig. 6. Robust design for faster response. (a) $y$ (solid), $y_{m}$ (dashed); (b) $u$.

quite different (although they satisfy the same performance specification). For one model in the uncertainty ellipse, the dominant poles are complex, thus, giving a response with overshoot. For another model in the uncertainty ellipse, the dominant pole is real and a monotonous response is expected. This behavior is also observed in Fig. 6a, where the upward step is monotonous while the downward step has an oscillation. The reestimated closed-loop poles are

$\lambda\left(A_{c}^{*}\left(\hat{\theta}_{c}, \eta(\hat{\theta}, \mathbf{P})\right)\right)=0.8270 \pm 0.1043 i$.

The unmodeled response standard deviation is $\operatorname{STD}\left(e_{u}\right)=0.1704 \times 10^{-4} \mathrm{~m}$. Note that this is larger than for the slow desired response $\left(0.10 \times 10^{-4} \mathrm{~m}\right)$. However, as illustrated below, further reduction of the unmodeled response standard deviation can be obtained by using the internal model principle.

\subsection{Minimizing the unmodeled response}

An extension to the single data set procedure Steps 1-7 is now illustrated. A second experiment is made using the robust controller from Step 7. An improved, but higherorder controller is then estimated via the internal model principle.

Steps 8 and 9 repeated experiment: Re-estimation of $\hat{\theta}$ and estimation of $\hat{\theta}_{Q}$

From the repeated experiment, shown in Fig. 4, the plant model is re-estimated:

$\hat{\theta}_{2}=\left(\begin{array}{ll}\hat{\beta} & \hat{\gamma}\end{array}\right)^{\mathrm{T}}=\left(\begin{array}{lll}2.0308 & 1.2366\end{array}\right)^{\mathrm{T}}$.

The subscript 2 is used here to indicate the second experiment. Notice that the estimated gain $\hat{\gamma}$ is $34 \%$ larger than the previous estimate from the first experiment (see (15), 

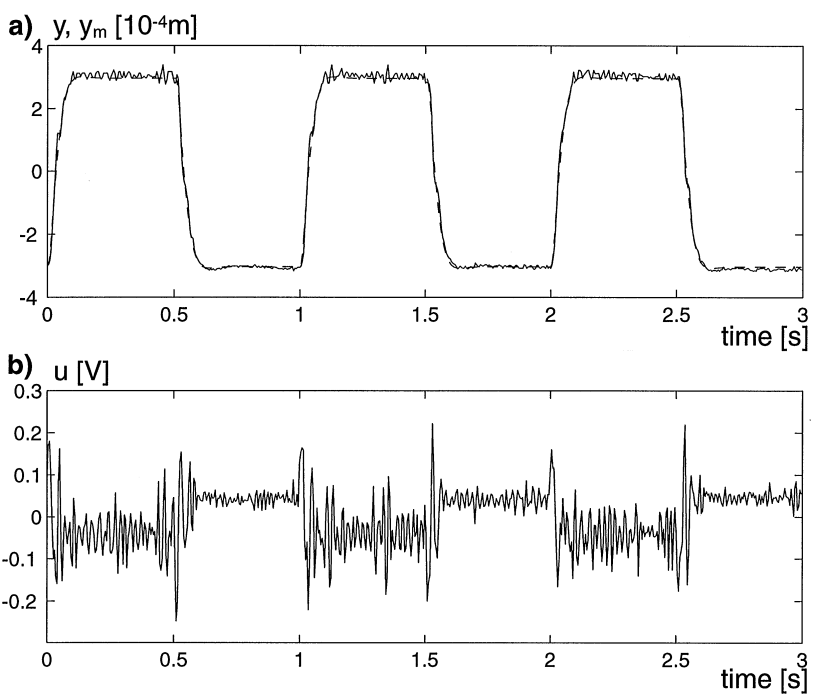

Fig. 7. Robust design for fast response based on the internal model principle. (a) $y$ (solid), $y_{m}$ (dashed); (b) $u$.

in the sequel denoted by $\hat{\theta}_{1}$ ). The new model, together with the robust controller from Step 7, gives

$$
\begin{aligned}
\lambda\left(\boldsymbol{A}_{c}\left(\hat{\theta}_{2}, \eta\left(\hat{\theta}_{1}, \mathbf{P}\right)\right)\right)= & 0.8284 \pm 0.0956 i, \\
& 0.3691 \pm 0.5607 i,-0.1674 .
\end{aligned}
$$

Clearly, $\hat{\theta}_{2}$ enables to predict the identified dominant closed-loop poles (17) better than $\hat{\theta}_{1}$ does (16). The internal model principle is now illustrated for the estimation of one $\left(\operatorname{dim} \theta_{Q}^{1}=1\right)$ and two $\left(\operatorname{dim} \theta_{Q}^{2}=2\right)$ parameters. In both cases $R_{f i x}=1$ and $S_{f i x}=1+q^{-1}$. The resulting controllers $\eta\left(\widehat{\theta}_{O}^{1}, \hat{\theta}_{2}\right)$ and $\eta\left(\hat{\theta}_{Q}^{2}, \hat{\theta}_{2}\right)$ are evaluated below.

For $\eta\left(\hat{\theta}_{Q}^{1}, \hat{\theta}_{2}\right)$, the estimated closed-loop poles are

$$
\lambda\left(A_{c}^{*}\left(\hat{\theta}_{c}, \eta\left(\widehat{\theta}_{Q}^{1}, \hat{\theta}_{2}\right)\right)\right)=0.8368,0.7020
$$

with the unmodeled response standard deviation $\operatorname{STD}\left(e_{u}\right)=0.1675 \times 10^{-4} \mathrm{~m}$. With $\operatorname{dim} \theta_{Q}^{1}=1, \operatorname{deg} Q=$ 0 , and the order of $R$ does not augment compared to the design in Step 7. Also, the dominant pole of $\lambda(R)$ is 0.96 , which corresponds to the choice of $R_{f i x}$ in Step 7. Therefore, the reduction of the unmodeled response is merely a result of the improved estimated plant model $\hat{\theta}_{2}$. However, the improvement is only $1.7 \%$ (of $0.1704 \times 10^{-4} \mathrm{~m}$ in Step 7).

For $\eta\left(\hat{\theta}_{Q}^{2}, \hat{\theta}_{2}\right)$, the estimated closed-loop poles are

$$
\lambda\left(A_{c}^{*}\left(\hat{\theta}_{c}, \eta\left(\hat{\theta}_{Q}^{2}, \hat{\theta}_{2}\right)\right)\right)=0.7821 \pm 0.0547 i \text {. }
$$

These are closer than (19) to the dominant designed closed-loop poles (18). Moreover, $\operatorname{STD}\left(e_{u}\right)=0.1386 \times$ $10^{-4} \mathrm{~m}$, a reduction of $18.66 \%$ compared to Step $7\left(0.1704 \times 10^{-4} \mathrm{~m}\right)$. The response is shown in Fig. 7a. Notice that the oscillations in the downward steps have disappeared. This can also be expected from Fig. $8 \mathrm{~b}$ showing that the dominant closed-loop poles are almost invariant for the uncertainty ellipse.
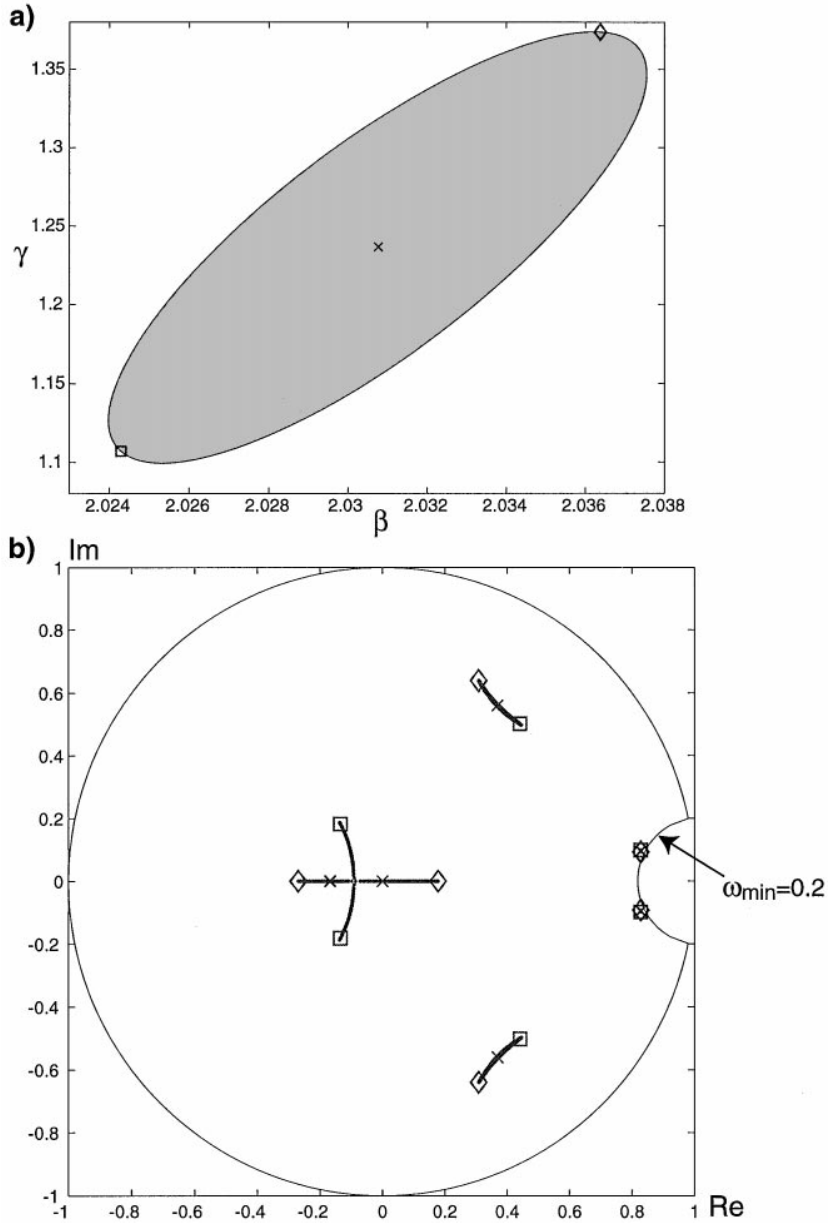

Fig. 8. Minimizing the unmodeled response. (a) Nominal model ' $x$ ' and $90 \%$ uncertainty ellipse; (b) closed-loop nominal poles ' $x$ ' and poles corresponding to the ellipse boundary (with special examples marked $\square$ and $\diamond)$.

Steps 8 and 9 show that there is a lot more to gain for the rejection of the unmodeled response by estimating the internal disturbance model rather than estimating the plant model.

\section{Conclusions}

A data-driven controller design procedure with robust performance has been developed and evaluated on a magnetic suspension process. The data are used to estimate, in closed-loop operation, the plant model as well as the model uncertainty.

A data filter is estimated first. There are two reasons for this: (i) it enables the subsequent use of a linear least-square technique, and (ii) it provides a straightforward means to validate the estimated model.

An ellipsoidal uncertainty bound in parameter space is estimated. A trade-off between robustness and performance is possible by specifying the sizes of the ellipsoid and of a generalized stability region, respectively. 
A simple generalized stability region, parameterized by specifying the desired speed and the relative damping, is used for illustration. The ellipsoidal robustness consideration turns out to be important and gives improved performance over a large operational range.

An extension of the robust performance procedure is also proposed, where a disturbance model describing the unmodeled response is estimated according to the internal model principle. This optimizes the rejection of the unmodeled response for a given reference signal. For the magnetic suspension system studied here, this is considerably more beneficial for reducing the unmodeled response standard deviation (19\% improvement) than one additional iteration involving estimating a new plant model and designing a new controller $(1.7 \%$ improvement).

\section{Acknowledgements}

This work has been financed by the Swiss National Foundation for Scientific Research under Project \# 2142316.94.

\section{References}

Åström, K. J. (1993). Matching criteria for control and identification. Proceedings of the 1993 European control conference, Groningen (pp. 248-251).
Bitmead, R. R. (1993). Iterative control design approaches. Prep. of 12th IFAC world congress, Sydney, Vol. 9 (pp. 381-384).

De Callafon, R., \& Van Den Hof, P. (1997). Suboptimal feedback control by a scheme of iterative identification and control design. Mathematical Modelling of Systems, 3(1), 77-101.

Gevers, M., \& Ljung, L. (1986). Optimal experiment designs with respect to the intended model application. Automatica, 22, 543-554.

Hjalmarsson, H., Gevers, M., De Bruyne, F., \& Leblond, J. (1996). For model-based control design, closed loop identification gives better performance. Automatica, 32(12), 1659-1673.

Holmberg, U., Myszkorowski, P., \& Bonvin, D. (1997). A pole-placement approach that matches identification and control objectives. Proceedings of the European control conference, Brussels, TH-A F6.

Holmberg, U., \& Valentinotti, S. (1997). Identification for control of a magnetic levitation system. Technical Report 1997.02, Institut d'automatique, EPFL, Switzerland.

Landau, I. D., \& Karimi, A. (1997). An output error recursive algorithm for unbiased identification in closed loop. Automatica, 33(5), 933-938.

Langer, J., \& Landau, I. (1996). Closed loop identification as a design tool for the robust digital control of a $360^{\circ}$ flexible arm. IFAC 13th triennial world congress, San Francisco (pp. 49-54) 1b-02 4.

Ljung, L. (1987). System identification - theory for the user, Englewood Cliffs, NJ: Prentice-Hall, 07632.

Rantzer, A., \& Megretski, A. (1994). A convex parameterization of robustly stabilizing controllers. IEEE Transactions on Automatic Control, 39(9), 1802-1808.

Raynaud, H. F. (1991). $H_{\infty}$-ellipsoidal controller — an approach to robustness with respect to parameter uncertainties. Proceedings of 1991 European control conference, Grenoble (pp. 2601-2604).

Tsypkin, Y. Z. (1991). Adaptive-invariant discrete control systems. In P. V. Kokotović, Foundations of adaptive control, lecture notes in control and information sciences (pp. 239-268) Berlin: Springer.

Van den Hof, P. M. J., \& Schrama, R. J. P. (1995). Identification and control - closed-loop issues. Automatica, 31, 1751-1770. 